\title{
Aharonov-Bohm scattering by vortices of dimensionally-reduced Yang-Mills field
}

\author{
Kiyoshi Shiraishi \\ Department of Physics, Faculty of Science, Ochanomizu University, \\ 1-1 Otsuka 2, Bunkyou-ku, Tokyo 112, Japan \\ and \\ Atsushi Nakamula \\ Department of Physics, Tokyo Metropolitan University, \\ Setagaya-ku, Tokyo 158, Japan \\ Czechoslovak Journal of Physics Volume 42, \\ Number 3 (1992), 285-289
}

\begin{abstract}
If two dimensions of six-dimensional space-time are compactified, a topological configuration of Yang-Mills gauge field appears as a cosmic string in four dimensions, whose thickness is of the same order as the size of the compact space. We consider scattering of low-energy fermions by this object.
\end{abstract}

Recently, the present authors and Hirenzaki have verified the existence of a vortex-like classical solution in a six-dimensional Yang-Mills (YM) system with partially compactified space [1]. This object appears to be a cosmic string (CS) [2] in our space-time. It is notable that this string arises without the need for elementary scalar fields. CSs of this type might appear in Kaluza-Klein models including the anomaly-free supergravity model proposed in ref. [3]. This CS may be connected with scenarios of making clusters of galaxies in (multidimensional) cosmology.

In ordinary CS scenarios of formation of a large scale structure, it is necessary to consider gravitational interactions of cosmic strings. Their characteristics have been understood by analytical and/or numerical methods. Interactions of matter with CS without gravity have also been investigated in various models, which were principally related to topics of proton decay in unified theories $[4,5]$.

The most peculiar interaction independent of details in models is known as the Aharonov-Bohm (AB) scattering [6]. The scattering of particles occur in the region far away from the axis of CS, where the gauge configuration is expressed as pure-gauge. This effect is due to phases of wave functions of the particles. 
In this letter, we consider the $\mathrm{AB}$ scattering in the $\mathrm{CS}$ model of our sixdimensional system [1]. The main distinction from the ordinary four-dimensional models mentioned above is that matter fields also come from six-dimensional matter fields. We must be careful of the fact that a particle with a unit charge is not scattered by a CS with a quantized flux (in the absence of gravity). This phenomenon is understood as the same situation of the "string" in the Dirac monopole [7]. For the simplest case, namely, in a $U(1)$-Higgs system, there is no scattering in the case with only a unit gauge coupling constant.

Since our model consists of YM field, no charged field coupled in arbitrary strength is allowed. In this paper we introduce fermions in a fundamental representation of $S U(2)$ as a matter field. As we will see later, the effective charge of this fermion is half of the unit when the space-time is reduced.

Now, at the beginning, we review the CS-like solution to six-dimensional YM equations in the case of a constant geometry.

For a constant background space-time geometry, we choose a sphere as an extra compactified two-space. Further we give the parametrization of the components on this extra space as follows,

$$
\begin{aligned}
& A_{\theta}=\frac{1}{2}\left(\begin{array}{cc}
0 & -i \Phi e^{-i \phi} \\
i \Phi^{*} e^{i \phi} & 0
\end{array}\right), \\
& A_{\phi}=-\frac{1}{2} \sin \theta\left(\begin{array}{cc}
0 & \Phi e^{-i \phi} \\
\Phi^{*} e^{i \phi} & 0
\end{array}\right)+\frac{1}{2}\left(\begin{array}{cc}
1-\cos \theta & 0 \\
0 & -(1-\cos \theta)
\end{array}\right),
\end{aligned}
$$

where $\theta$ and $\phi$ are the standard spherical coordinates and $\Phi$ is assumed to depend only on the four-dimensional coordinates. Besides these components, we take out the following " $U(1)$ " part as the four-dimensional components of gauge field

$$
A_{\mu}=A_{\mu} \times \frac{1}{2}\left(\begin{array}{cc}
1 & 0 \\
0 & -1
\end{array}\right),
$$

By these parametrizations, the YM equations can be shown to be reduced to those of the Abelian-Higgs model in which $A_{\mu}$ and $\Phi$ are regarded as the $U(1)$ gauge field and the Higgs field, respectively [1]. In these equations the effective Higgs self-coupling is related with the gauge coupling constant.

Moreover the relation between the $U(1)$ gauge coupling and the Higgs coupling constant causes that a vortex solution is given as a self-dual solution to first-order simultaneous differential equations. Of course, this vortex solution is self-dual configuration of YM field in the original form. Namely, the classical solution represents embedding of an instanton into the four-space which consists of extra space plus the $x-y$ plane. The width of this string is of the same order of size as the extra space.

The classical solution with $n$-vorticity centered at the origin has the following behaviour:

$$
\begin{aligned}
& \Phi=0 \text { at the origin, } \Phi=e^{i n \varphi} \text { at the spatial infinity, } \\
& A_{\varphi}=0 \text { at the origin, } A_{\varphi}=-n \text { at the spatial infinity, }
\end{aligned}
$$


where $r$ and $\varphi$ are the polar coordinates of $x-y$ plane. In this description we do not use the orthonormal coordinate basis but a general coordinate basis. If $r$ is infinity, the field strengths of $S U(2)$ are zero, i.e., expressed in a pure-gauge form. The magnitude of flux is determined by the winding number, $n$.

It is important to note that the flux of the string is not arbitrary as in usual cosmic string models.

Now, we consider a fermion in the fundamental representation of $S U(2)$ as a matter field. In six dimensions, a Dirac fermion has at least eight complex components. Here, for simplicity, we deal with a massless Weyl fermion, while an extension to a general case is straightforward. The Dirac matrices in six dimensions are defined as follows;

$$
\begin{aligned}
& \Gamma^{\mu}=\gamma^{\mu} \otimes \sigma^{1}, \quad(\mu=0,1,2,3), \\
& \Gamma^{4}=\gamma_{5} \otimes \sigma^{1}, \\
& \Gamma^{5}=1_{4} \otimes \sigma^{2},
\end{aligned}
$$

where $\gamma^{\mu}, \gamma_{5}$ and $1_{4}$ are the four-dimensional Dirac matrices, chiral and unit matrices, respectively. The Weyl condition is $\Gamma_{7} \Psi=\gamma_{5} \otimes \sigma^{3} \Psi=+\Psi$.

When the compact space has non-zero curvature, the fermions acquire super heavy masses in four dimensions. This is also true when the fermions are coupled only with the gauge field which is expressed merely by pure gauge. In the present model, we can have massless particle states by introducing a coupling with a topologically non-trivial gauge field configuration [8]. In ref. [1], we have introduced an extra $U(1)$ gauge field to stabilize the compact space. Here we make use of it to obtain massless modes of fermion.

The Dirac equation is written as follows,

$$
\Gamma^{M} D_{M} \Psi=\Gamma^{M}\left(\partial_{M}+\frac{1}{4} \omega_{M A B} \Gamma^{A B}+i A_{M}+i B_{M}\right) \Psi=0,
$$

where $\Gamma^{A B}=\frac{1}{2}\left[\Gamma^{A}, \Gamma^{B}\right]$ and $B_{M}$ is the newly introduced $U(1)$ gauge field which has the following monopole-like classical configuration

$$
B_{M}=\frac{1}{2}(\cos \theta \pm 1) \text {. }
$$

We consider eigenstates of four-dimensional chiral matrix as $\gamma_{5} \psi=\psi$. We assume the string is sufficiently narrow in comparison with the wavelength of the matter field. We seek for the lowest energy states in (outside) vacuum, i.e., the zero modes of the covariant derivative which acts in the extra space. Other fermionic modes besides the zero mode have masses of the order of an inverse of the scale of the extra space, in our world.

There exist two of such zero-mode eigenfunctions which correspond to two components of the fundamental representation. Those are the following ones,

$$
\begin{aligned}
\Psi_{I}(\theta, \phi, \varphi) & =\left(\begin{array}{c}
\cos \frac{1}{2} \theta e^{i \phi / 2} \\
e^{-i n \varphi} \sin \frac{1}{2} \theta e^{3 i \phi / 2}
\end{array}\right), \\
\Psi_{I I}(\theta, \phi, \varphi) & =\left(\begin{array}{c}
-\sin \frac{1}{2} \theta e^{-i \phi / 2} \\
e^{-i n \varphi} \cos \frac{1}{2} \theta e^{i \phi / 2}
\end{array}\right),
\end{aligned}
$$


In these descriptions, the column denotes the components of $S U(2)$ doublet and we have omitted to write the eigenfunction $\psi$ of four-dimensional chiral matrix, i.e., $\gamma_{5} \psi=\psi$.

It is an essential difference from the eigenfunctions of ordinary Kaluza-Klein mode function that they depend on the azimuthal coordinate $\varphi$.

From now on, we can trace the same way as in ref. [5]. The whole eigenfunctions are given as direct products of what was derived above and of the following wave function

$$
P_{k l}^{( \pm)}(r, \varphi)=a_{k}\left(\begin{array}{c}
J_{ \pm(l+n / 2)}(k r) \\
\pm i J_{ \pm(l+n / 2+1)}(k r) e^{i \varphi}
\end{array}\right) e^{i l \varphi-i \omega t},
$$

where $J_{n}(x)$ 's are the Bessel functions, $k$ is the energy of the partial wave and $l$ is the label of the angular momentum. The two components here are those of a Weyl fermion.

In the approximation of infinitesimal string thickness, we can get the differential cross section by the standard way [5] with the assumption that the functional form of the extra-dimensional portion of incoming and outgoing waves are the same. The cross section per unit length (along the string) is,

$$
\frac{d \sigma}{d \Theta}=\frac{\frac{1}{2}\left[1-(-1)^{n}\right]}{2 \pi k \sin ^{2} \frac{\Theta}{2}}
$$

where $\Theta$ is a scattering angle.

As a conclusion of this, we can observe scattering of a massless fermion in the fundamental representation of $S U(2)$ by the string which has the flux with any odd-numbered unit, in our model.

We must emphasize that in our model the charge of the fermions differs by a factor $\frac{1}{2}$ from the usual one, giving a scattering cross section with maximal Aharonov-Bohm enhancement. The cause of this is that the coupling constant between $A_{\mu}$ and $\Psi$ is half of that between $A_{\mu}$ and $\Phi$. The factor $\frac{1}{2}$ can be seen in equation (3).

This model will be extended to have a greater group and a higher-dimensional extra space. In such a case it is expected that there occurs the AB scattering by the CS of the present kind. Obviously, matter fields in the faithful representations of the group, such as the adjoint representation, are never scattered by the CS.

In the case that the wavelength is comparable with the string thickness, namely when the thickness cannot be negligible, we should treat the wave function inside the string core exactly. Then we analyze whether mixing among Kaluza-Klein excitation states occurs or not. Since dependence in extra-dimensional coordinates of eigenfunctions varies with the four-dimensional coordinate $r$, it is suitable to study the effects of extra space in a very early, multi-dimensional, universe. Further, it is a challenging problem for numerical analysis. This will be a future subject. 
The authors would like to thank S. Hirenzaki for some useful comments. One of the authors (KS) would like to thank A. Sugamoto for reading this manuscript. KS is indebted to Soryuusi shogakukai for financial support. He also would like to acknowledge the financial aid of Iwanami Füjukai.

\section{References}

[1] Nakamula A., Hirenzaki S., Shiraishi K.: Nucl. Phys. B339 (1990) 533.

Nakamula A., Shiraishi K.: Mod. Phys. Lett. A5 (1990) 1109.

Nakamula A., Shiraishi K.: Prog. Theor. Phys. 84 (1990) 1100.

[2] Vilenkin A.: Phys. Rep.121 (1985) 263.

[3] Randjbar-Daemi S., Salam A., Sezgin E., Strathdee J.: Phys. Lett. B151 (1985) 351.

[4] Brandenberger R. H., Perivolaropoulos L.: Phys. Lett. B205 (1988) 396.

Brandenberger R. H., Davis A. C., Matheson A. M.: Nucl. Phys. B307 (1988) 909; Phys. Lett. B218 (1989) 304.

Davis A. C., Perkins W.: Phys. Lett. B218 (1989) 37.

Gregory R., Davis A. C., Brandenberger R. H.: Nucl. Phys. B323 (1989) 187.

Perivolaropoulos L., Matheson A. M., Davis A. C., Brandenberger R. H.: Phys. Lett. B245 (1990) 556.

Matheson A. M., Perivolaropoulos L., Perkins W., Davis A. C., Brandenberger R. H.: Phys. Lett. B248 (1990) 263.

Alford M. G., March-Russell J., Wilczek F.: Nucl. Phys. B328 (1989) 140.

Kogan Ya. I., Selivanov K. G.: Zh. Eksp. Teor. Fiz. 97 (1990) 387.

de Sousa Gerbert Ph.: Phys. Rev. D40 (1989) 1346.

[5] Alford M. G., Wilczek F.: Phys. Rev. Lett. 62 (1989) 1071.

[6] Aharonov Y., Bohm D.: Phys. Rev. 115 (1959) 485.

[7] Dirac P. A. M.: Proc. R. Soc. London A133 (1931) 60.

[8] Randjbar-Daemi S., Salam A., Strathdee J.: Nucl. Phys. B214 (1983) 491. 